\title{
A NEW HIGH RESOLUTION OPTICAL METHOD FOR OBTAINING THE TOPOGRAPHY OF FRACTURE SURFACES IN ROCKS
}

\author{
Steven Ogilvie, Evgeny IsAKov, Colin TAYlor AND PAul Glover \\ Department of Geology and Petroleum Geology, University of Aberdeen, Aberdeen AB24 3UE, Scotland \\ e-mail: s.ogilvie@abdn.ac.uk, e.isakov@abdn.ac.uk, c.w.taylor@abdn.ac.uk, p.glover@abdn.ac.uk \\ (Accepted February 21, 2002)
}

\begin{abstract}
Surface roughness plays a major role in the movement of fluids through fracture systems. Fracture surface profiling is necessary to tune the properties of numerical fractures required in fluid flow modelling to those of real rock fractures. This is achieved using a variety of (i) mechanical and (ii) optical techniques. Stylus profilometry is a popularly used mechanical method and can measure surface heights with high precision, but only gives a good horizontal resolution in one direction on the fracture plane. This method is also expensive and simultaneous coverage of the surface is not possible. Here, we describe the development of an optical method which images cast copies of rough rock fractures using in-house developed hardware and image analysis software (OptiProf ${ }^{\mathrm{TM}}$ ) that incorporates image improvement and noise suppression features. This technique images at high resolutions, $15-200 \mu \mathrm{m}$ for imaged areas of $10 \times 7.5 \mathrm{~mm}$ and $100 \times 133 \mathrm{~mm}$, respectively and a similar vertical resolution $(15 \mu \mathrm{m})$ for a maximum topography of $4 \mathrm{~mm}$. It uses in-house developed hardware and image analysis (OptiProf ${ }^{\mathrm{TM}}$ ) software and is cheap and non-destructive, providing continuous coverage of the fracture surface. The fracture models are covered with dye and fluid thicknesses above the rough surfaces converted into topographies using the Lambert-Beer Law. The dye is calibrated using 2 devices with accurately known thickness; (i) a polycarbonate tile with wells of different depths and (ii) a wedge-shaped vial made from silica glass. The data from each of the two surfaces can be combined to provide an aperture map of the fracture for the scenario where the surfaces touch at a single point or any greater mean aperture. The topography and aperture maps are used to provide data for the generation of synthetic fractures, tuned to the original fracture and used in numerical flow modelling.
\end{abstract}

Keywords: fracture topography, image acquisition, optical imaging.

\section{INTRODUCTION}

Surface roughness has a large influence upon fluid flow through fracture systems (Isakov et al., 2001a). Accurate surface parameterisation is required for incorporation of realistic fracture roughness into models of fluid flow for rough fractures. Synthetic fractures can then be created which are tuned to share these features (Isakov et al., 2001b). Only then can these numerical fractures be used for modelling fluid flow using the local cubic-law, or by solution of the Reynold's or Navier Stoke's equations (Zimmerman and Yeo, 2000).

Surface data acquisition techniques fall into two main categories; (i) optical and (ii) mechanical, both of which are comprehensively reviewed in the literature (e.g., Adler and Thovert, 1999; Develi et al., 2001). Mechanical methods such as stylus profilometry (e.g., Brown and Scholz, 1985) can measure surface heights with high precision, but only give a good horizontal resolution in one direction $(c .0 .02 \mu \mathrm{m})$ on the fracture plane. The resolution in the other direction can be greater than $1000 \mu \mathrm{m}$. Furthermore, this method is expensive and simultaneous coverage of the surface is not possible. Laser profilometry uses a laser instead of a mechanical needle, and interferometry of the reflected light to measure the height of the surface (Voss and Shotwell, 1990). Counter-intuitively, perhaps the laser profilometer has a worse resolution than the needle/mechanical method. It also suffers the same profiling and alignment problems as the mechanical profilometer.

This has motivated our development of a nondestructive optical method (Isakov et al., 2001a), which provides continuous coverage of cast copies of rough rock fractures $(x y$-size $120 \times 120 \mathrm{~mm})$ at high resolutions $(15-200 \mu \mathrm{m}$ for imaged areas of $10 \times 7.5$ $\mathrm{mm}$ and $100 \times 133 \mathrm{~mm}$, respectively) using in-house developed hardware and image analysis (OptiProf ${ }^{\mathrm{TM}}$ ) software. For a maximum topography of $4 \mathrm{~mm}$, the 
vertical resolution is $15 \mu \mathrm{m}$. Such spectrophotometric analysis (SA) of fracture surfaces has up to 1 order of magnitude greater spatial resolution than nuclear magnetic resonance (NMR) or computerised tomography (CT) scanning of real fracture surfaces (Renshaw et al., 2000).

There are several technical difficulties to overcome in the imaging process including dynamic noise in light source and video stream and static distortions in the video signal. These have been resolved by OptiProf ${ }^{\mathrm{TM}}$ software that (i) calibrates the imaging system, (ii) controls the capture of images, (iii) makes appropriate corrections, and (iv) calculates the final measured topography of the surface.

Statistical analysis of these surfaces is carried out using in-house software ParaFrac ${ }^{\mathrm{TM}}$, which feeds SynFrac ${ }^{\mathrm{TM}}$ software, to create numerical fractures tuned to contain these properties (Isakov et al., 2001b).

\section{METHODS AND MEASUREMENT}

\section{MATERIALS}

Five rock samples were trimmed to form blocks with $120 \times 120 \times 100 \mathrm{~mm}$ nominal dimensions. Rough fractures were artificially created by stress exposure around the sample block. Each fracture half was taken, cleaned with compressed air and placed on a glass working surface (Fig. 1). Thin polycarbonate walls were added to the sides of the sample and Silastic ERTV $^{\circledR}$ was poured over the surface. The mixture then cures to a white rubber with negligible shrinkage and exothermic heating, allowing the complex structure to be accurately reproduced without damage by thermal stresses. The rubber was pealed off the surface and placed, rough surface uppermost, on the glass surface and surrounded by walls. Bondaglass ${ }^{\circledR}$ clear casting resin was poured in on top of the Silastic peal and allowed to set. The cast HFPM was then trimmed to $100 \times 100 \times 30 \mathrm{~mm}$ and polished. The quality of reproduction of this technique is illustrated in Fig. 2. Scanning electron microscope (SEM) images of original rock fracture (a) and cast (b) illustrate a very high fidelity of reproduction. In fact, detailed visual inspection of the majority of the area covered by the SEM images reveals that the HFPM is reproduced to within $1 \mu \mathrm{m}$. However, reproduction in certain areas (i.e., centre top in Fig. 2b) is affected by (i) minerals dislodged during the casting process, (ii) dust and other particles, and (iii) bubbles.

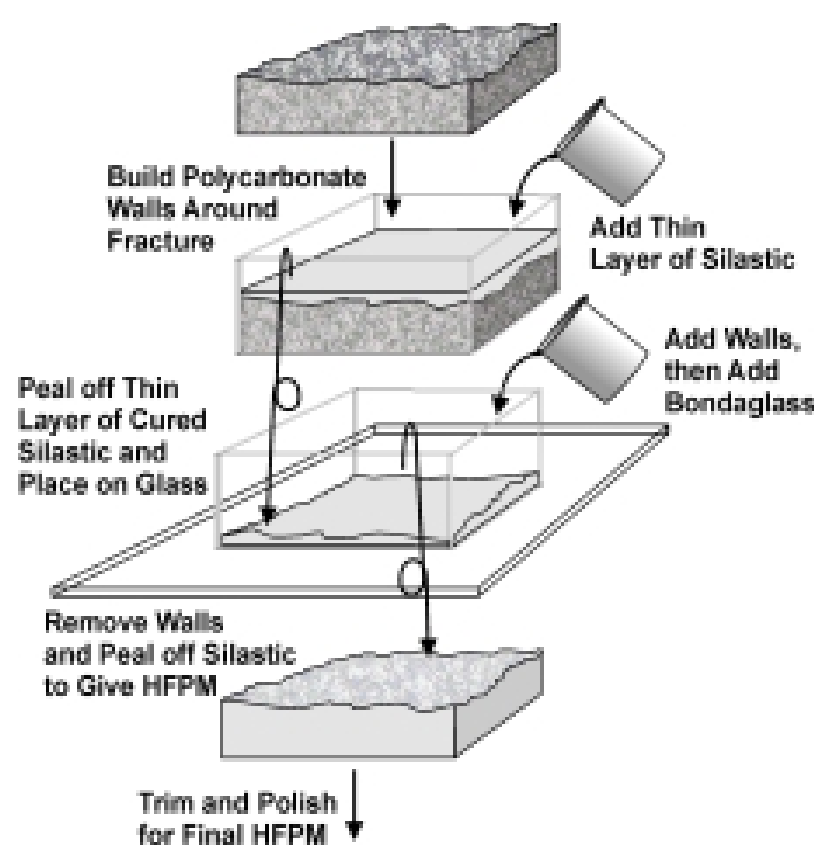

Fig. 1. Preparation of High Fidelity Polymer Models (HFPMs) from rock fracture surfaces.

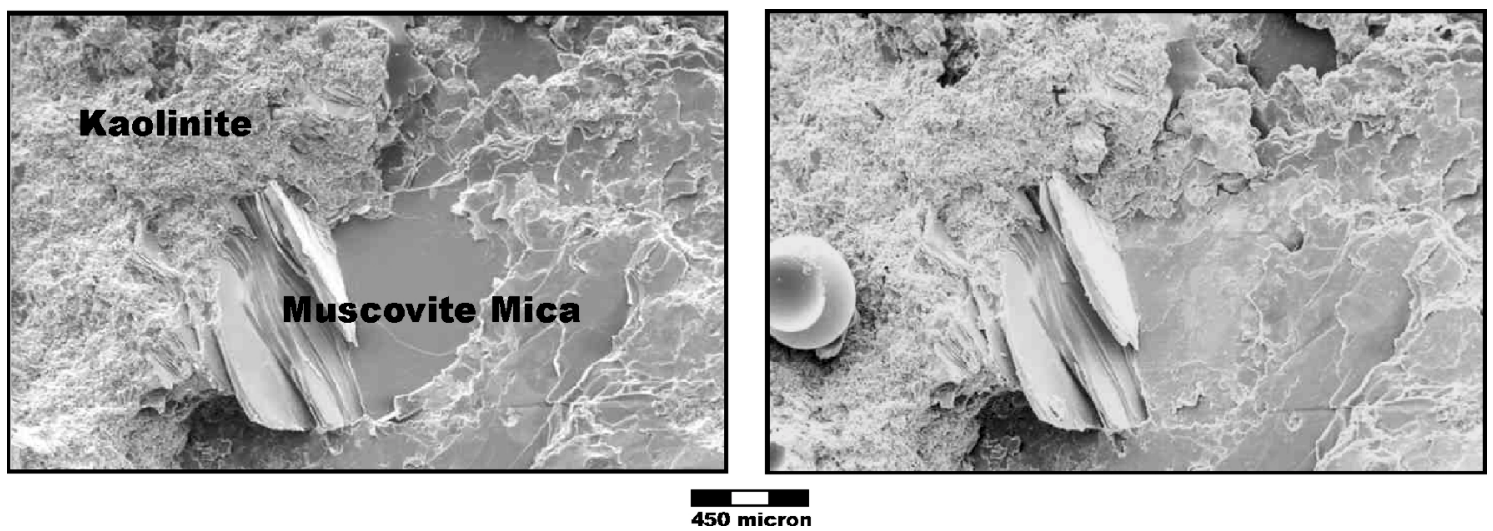

(a)

(b)

Fig. 2. The quality of reproduction of fracture surfaces by HFPMs. (a) SEM (secondary electron) image of the surface of the original rock and (b) exactly corresponding area of the resulting HFPM. A gas bubble in (b) usefully distinguishes between the two images. 


\section{DEVICES}

Each of the HFPMs was subjected to digital optical imaging (Fig. 3). Thin polycarbonate walls were built up around the sides of each HFPM (Fig. $3 a)$ and placed on a light box under a digital colour camera $(640 \times 480$ pixels, 8 -bit grey-scale depth), which was attached to a PC equipped with a video capture board (Fig. 3b). The HFPM was imaged 20 times, first while containing distilled water, and then while containing the same amount of dyed water. These images represent the extent to which the incident intensity of light is absorbed by the presence of the HFPM and the fluid covering the surface. The ratio of the intensity of light for a given pixel at a given location on the fracture between the images containing dye and those containing water is related to the thickness of fluid covering the rough surface. This is described by the Lambert-Beer Law,

$$
I_{x}=I_{o} e^{-K c T}
$$

where, $I_{x}$ is the intensity of the transmitted light, $I_{o}$ is the intensity of the incident light, $K$ is a material dependent property describing the efficiency with which a material adsorbs light, $c$ is the concentration of the material, and $T$ is the thickness of the material through which the light has passed.

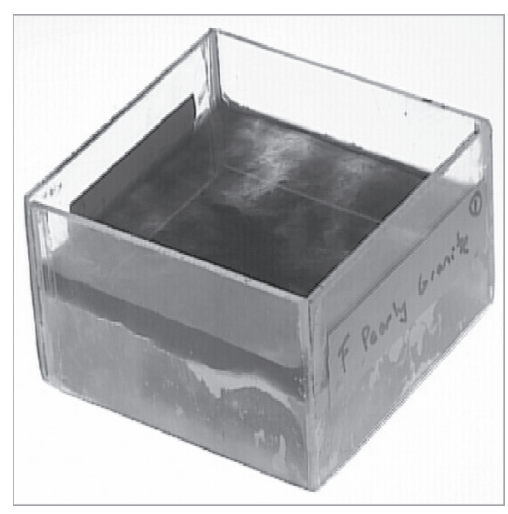

(a)

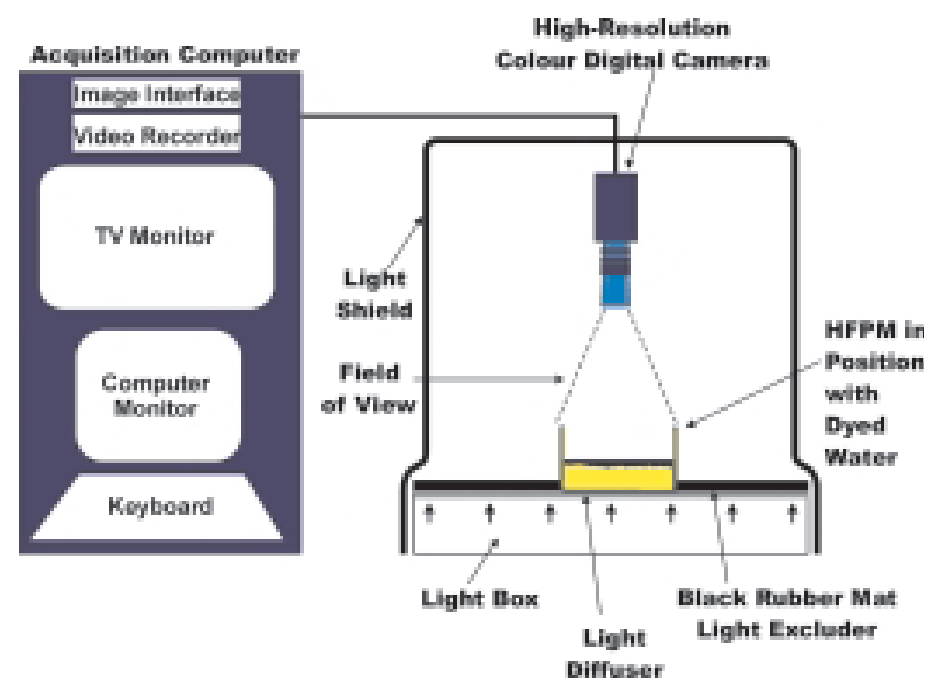

(b)

Fig. 3. Digital optical imaging setup. (a) HFPM surrounded by polycarbonate walls and filled with dye to be imaged by digital optical imaging equipment (b) Here, the camera captures images of the HFPM covered with water, then dyed water and the images are processed using image analysis software (to the left).

\section{CALIBRATION}

The fluid thickness is calculated from the measured intensity ratio by experimental calibration of dyed and undyed fluids, which provides a measurement of the light extinction properties of the dye. This was carried out using two devices with accurately known fluid thicknesses (Fig. 4); (i) a polycarbonate tile with 9 wells with depths from $0.25 \mathrm{~mm}$ to $4.00 \mathrm{~mm}$, (ii) a wedge-shaped vial made from silica glass (aperture varies linearly from $0.00 \mathrm{~mm}$ to $4.30 \mathrm{~mm}$ ). Optical similarity between the HFPM material and the devices is not required. This is because the ratio of intensity of light passing through the subject filled with water to the intensity of light passing through the subject filled with dyed water depends upon depth and lightabsorbing properties of the dye only.

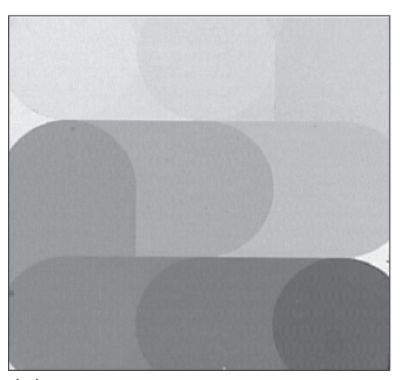

(a)

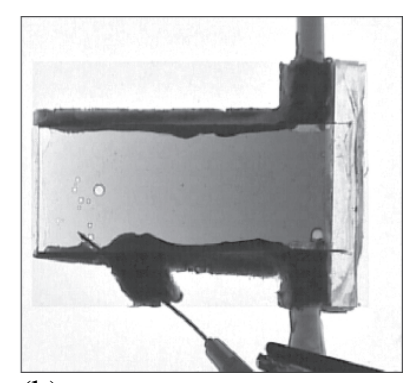

(b)
Fig. 4. Calibration devices used to calculate the topography of rough surfaces. (a) polycarbonate tile with 9 pockets of known thickness and (b) secondary tile device with thickness variation from 0 on the left to $4.3 \mathrm{~mm}$ on the right. 
Each pocket of the tile was filled with undyed water. The tile was then imaged, producing 8-bit greyscale images, intensities varying from 0 to 255 (Fig. 4a), and a clearfield equalization was performed to remove any spatial variations in incident light intensity from the image. The clearfield is an image of the background without any subject and is subtracted from the image of the tile using SigmaScan Pro $5^{\circledR}$ to correct the average image of the tile. The corrected image was divided pixel by pixel by that of the dyed water. The resulting image was analysed in SigmaScan Pro $5^{\circledR}$ to obtain the number of pixels present in each well for each intensity value, 0 to 255 (Fig. 5a). Gaussian curves were fitted to this data to obtain the mean intensity value and the standard deviation in intensity for each well. A calibration curve was constructed of intensity ratio as a function of fluid thickness from the data for all nine wells including error bars representing the standard deviations of intensity ratio and thickness (Fig. 5b). A similar process was carried out for the wedge filled with both dyed and undyed water (Fig. 4b). As the wedge provides a continuous variation of thicknesses and dye intensities, it was possible to obtain 440 data point pairs of intensity/thickness values (Fig. 5b). The tile calibration and the wedge calibration are in close agreement, and the standard deviation of the tile intensity data, well constrains the wedge data. This calibration curve is linear on the log-lin scales used in this diagram and therefore conforms to the LambertBeer Law.

Knowledge of $K_{d}$ (extinction coefficient of the dye) and $c_{d}$ (concentration of dye) allows the thickness of dye $T$ to be obtained from the intensities of light measured. While we know $c_{d}$ for each fluid, $K_{d}$ is unknown, hence we experimentally calibrate the intensity ratio $I_{d y e} / I_{\text {water }}$ as a function of fluid thickness $T$. This process provides the value of $K_{d}$ for the dye. Once known, the thickness of dye $\mathrm{T}$ above a rough surface can be calculated for the measured light intensities and the values of $K_{d}$ and $C_{d}$, thus,

$$
T=\frac{\log \left(I_{\text {water }}\right)-\log \left(I_{d y e}\right)}{K_{d} c_{d}} .
$$

\section{CORRECTIONS}

The individual fracture surfaces are imaged while they are covered with first the dyed and then the undyed water. These images are then calibrated using the data from the procedure already described to provide a map of the fluid thickness above the rough surface topography. The resulting data can be transformed simply to provide a fully determined topography for each surface, and data from each of two surfaces can be combined to provide an aperture map of the fracture for the scenario where the surfaces touch at a single point or any greater mean aperture. There are, however, several technical difficulties to be overcome during the imaging process which are corrected for by the OptiProf ${ }^{\mathrm{TM}}$ software. The variation in some of these features is small, but noticeable, and will contribute to errors in the final calculated heights of the surface and hence aperture, if not corrected for (Table 1).

Each of the of the averaged intensity images from the measurements on the undyed and dyed water were subjected to a clearfield equalisation by OptiProf ${ }^{\mathrm{TM}}$, during which process any variations due to changes in the incident light source intensity are removed. The clearfield was obtained by taking multiple images of the light source, and averaging them to remove dynamic noise.
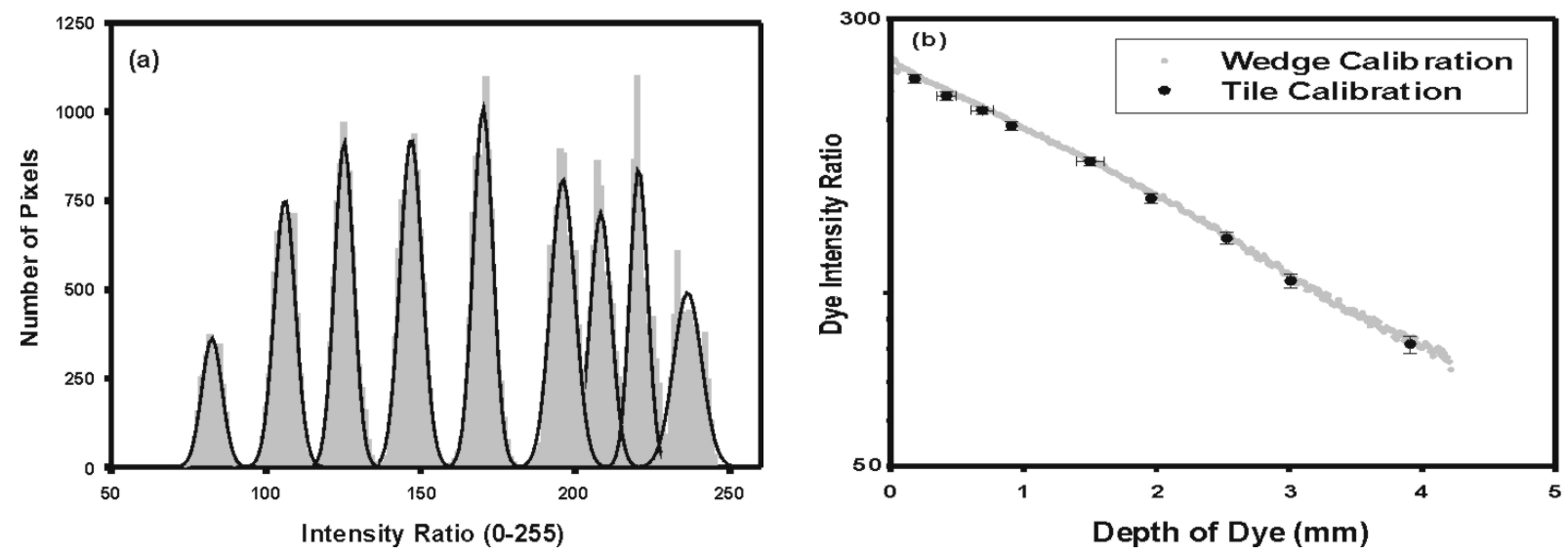

Fig. 5. Calibration results. (a) Individual pockets of the tile analysed for intensity distributions (b) Ratio of intensity of images for HFPMs containing dye to those containing water against depth of dye. The errors in this data are well constrained by scatter in the wedge data. Note the slight non-linearity in the wedge data due to slight curvature in the glass plates used to make the wedge. 
Table 1. Technical difficulties encountered during the imaging process and their solutions, which are incorporated into OptiProf ${ }^{T M}$ software.

\begin{tabular}{|c|c|}
\hline Technical Difficulty & Solution \\
\hline $\begin{array}{l}\text { 1. Fluid Level Control: Each of the fractures must be filled } \\
\text { with dyed and undyed water up to exactly the same arbitrary } \\
\text { level. }\end{array}$ & $\begin{array}{l}\text { A horizontal datum line is marked onto opposite walls } \\
\text { surrounding the fracture. Lining these up and filling to this } \\
\text { level removes the parallax errors. }\end{array}$ \\
\hline $\begin{array}{l}\text { 2. Lateral alignment: The imaged surface must be in exactly } \\
\text { the same } x y \text { position for imaging with dyed water and } \\
\text { undyed water even though it must be moved for replacing } \\
\text { the fluids. }\end{array}$ & $\begin{array}{l}\text { Four fixed reference points within the software are set over } \\
\text { point marks that are etched into the top of the walls } \\
\text { surrounding the fracture These are used to realign the } \\
\text { HFPM when removed and refilled. }\end{array}$ \\
\hline $\begin{array}{l}\text { 3. Dynamic noise in the imaged light intensity: From the } \\
\text { light source and video stream. Leads to variations in } \\
\text { brightness of the imaged intensities. }\end{array}$ & $\begin{array}{l}\text { Taking multiple images of the HFPM with each fluid in } \\
\text { place, and averaging the result pixel by pixel }\end{array}$ \\
\hline $\begin{array}{l}\text { 4. Static noise in video signal: A stripe effect on an image of } \\
\text { a uniform field, which varies with camera lens aperture. }\end{array}$ & $\begin{array}{l}\text { The variation in the sensitivity of the CCD between each } \\
\text { pixel on its surface was removed by calibrating each pixel } \\
\text { of the CCD individually, for every aperture. }\end{array}$ \\
\hline 5. Non-uniformity of the light source & A clearfield equalization was performed. \\
\hline $\begin{array}{l}\text { 6. Bubbles and dust in the fluids: Particles and bubbles in } \\
\text { the fluids are mobile if the fluid is perturbed. }\end{array}$ & $\begin{array}{l}\text { The software compares multiple images with bubbles and } \\
\text { dust in different locations and recognizes characteristics } \\
\text { which move. These are removed from the relevant images } \\
\text { prior to averaging. }\end{array}$ \\
\hline $\begin{array}{l}\text { 7. Opaque particles in the HFPM: Small and uncommon. } \\
\text { Obvious in the final image as thin low intensity spikes. }\end{array}$ & $\begin{array}{l}\text { Recognized and removed with the affected pixel being } \\
\text { reduced to the weighted mean of the surrounding } 8 \text { pixels. }\end{array}$ \\
\hline
\end{tabular}

\section{RESULTS}

Cast replicas of a suite of Mode I (splitting) rock fractures have been imaged with the new technique. Profiling results from a sample of sandstone are shown on Fig. 6. Included are photographs of both fracture surfaces $(\mathrm{a}, \mathrm{c})$, the measured surface topographies (b,d) and the aperture map (e). The aperture is calculated when opposite surfaces touch at a single point. In each case the profiles are very accurate representations of the fracture surfaces. There are some scattered spikes in the profiles, which may be bubble artefacts in the high fidelity polymer models, (HFPMs), which were not considered to influence the profiling data and therefore no attempt was made to remove them.

\section{DISCUSSION AND CONCLUSIONS}

A new, fast and inexpensive method has been developed that allows the numerical determination of the surface topography of rough fractures (or any rough surface). The data from each of the two surfaces can be combined to provide an aperture map of the fracture for the scenario where the surface touch at a single point or any greater mean aperture.
We are currently working on more realistic mechanical scenarios.

This method has a high lateral resolution, which is $15-200 \mu \mathrm{m}$ for imaged areas of $10 \times 7.5 \mathrm{~mm}$ and $100 \times 133 \mathrm{~mm}$, respectively but can be better than this if higher resolution cameras are used. The method has a similar height resolution $(15 \mu \mathrm{m})$ for our set-up, but again could be much smaller and much better if 16-bit $(62.5 \mathrm{~nm})$ or 24-bit $(0.25 \mathrm{~nm})$ imaging hardware is used. The method relies on the calibration of a dyed fluid, which obeys the LambertBeer Law, and has been successfully tested upon a range of real rock fractures. High fidelity polymer models (HFPMs) of the rock fractures are used which are reproduced to within $1 \mu \mathrm{m}$. Our method, using a dye concentration of $1 \mathrm{~g} / \mathrm{l}$ calibrates for an aperture height range of $4 \mathrm{~mm}$ but an estimated range of $6 \mathrm{~mm}$ could be achieved by linear extrapolation of the data (Fig. 5b). Other technical developments built into OptiProf $^{\mathrm{TM}}$ software, include (i) multiple imaging, (ii) clearfield equalisation, (iii) stacking, (iv) bubble detection, (v) static detection, (vi) individual pixel calibration and (vii) precise filling. OptiProf ${ }^{\mathrm{TM}}$ is also used to calculate the final measured topography of the surface. ParaFrac ${ }^{\mathrm{TM}}$ statistically analyses the fracture surfaces and aperture for (i) probability densities of surface heights, (ii) fractal dimension and 
(iii) matching properties. SynFrac ${ }^{\mathrm{TM}}$ software produces any possible combination of synthetic fractures, tuned to this data but having differing physical topographies (Isakov et al., 2001b). The modelled apertures together with experimental flow data are input into twodimensional flow models (Ogilvie et al., 2001).

This work was funded by the Natural Environmental Research Council of the UK, as part of the Micro-to-Macro Thematic Programme.

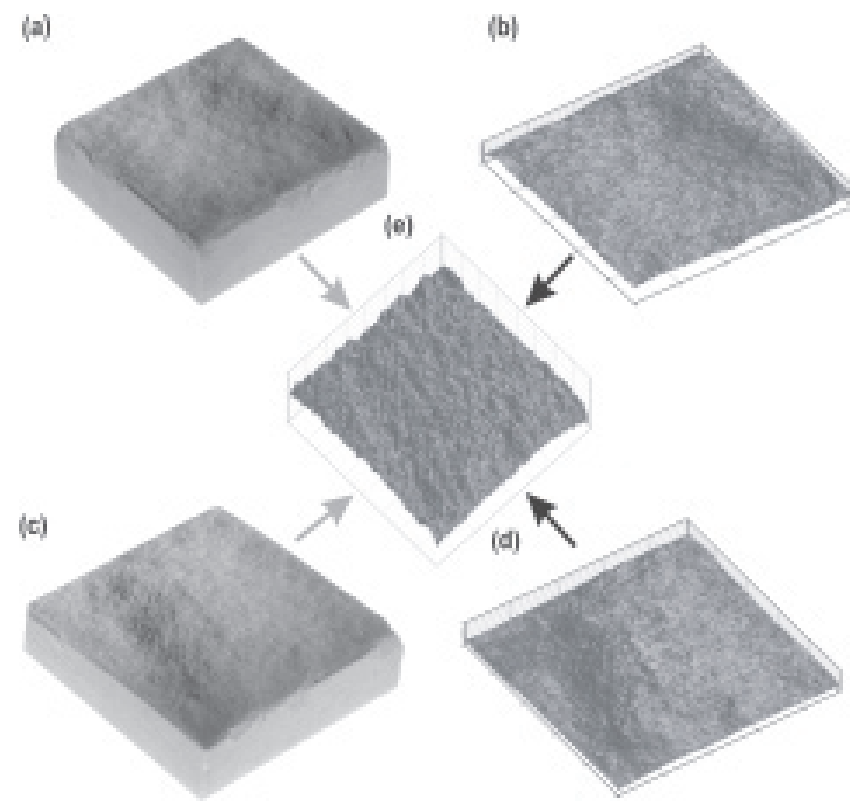

Fig. 6. Both fracture surfaces of a sandstone sample $(a, c)$, respective optical profiles $(b, d)$ and aperture map (e) produced using OptiProf ${ }^{T M}$ Surface dimensions $=100 \times 100 \times 4 \mathrm{~mm}$.

\section{REFERENCES}

Adler PM, Thovert JF (1999). Theory and Applications of Transport in Porous Media: Fractures and Fracture Networks. Kluwer Academic Publishers, 429.

Brown SR, Scholz CH (1985). Broad bandwidth study of the topography of natural rock surfaces. J Geophys Res 90:12575-82.

Develi K, Babadagli T, Comlekci C (2001). A new computer-controlled surface-scanning device for measurement of fracture surface roughness. Computers and Geosciences 27:265-77.

Isakov E, Ogilvie SR, Taylor CW, Glover PWJ (2001a). Fluid flow through rough fractures in rocks I: high resolution aperture determinations. Earth Plan Sci Lett 191:267-82.

Isakov E, Glover P, Ogilvie SR (2001b). Use of Synthetic Fractures in the Analysis of Natural Fracture Apertures Proceedings of the 8th European Congress for Stereology and Image Analysis, Image Analysis and Stereology, 20 (2) SUPPL. 1, Sept, 366-71.

Ogilvie, SR, Isakov, E, Glover, PWJ, Taylor, CW (2001). Use of Image Analysis and Finite Element Analysis to Characterise Fluid Flow in Rough Rock Fractures and their Synthetic Analogues. Proceedings of the 8th European Congress for Stereology and Image Analysis. Image Anal Stereol 20(2) Suppl 1, Sept, 504-9.

Renshaw CE, Dadakis JS, Brown SR (2000). Measuring fracture apertures: A comparison of methods. Geophys Res Lett 27(2):289-92.

Voss CF, Shotwell LR (1990). An investigation of the mechanical and hydraulic behaviour of tuff fractures under saturated conditions. In: High Level Radioactive Waste Management. La Grange Park III. American Nuclear Society, 825-34.

Zimmerman RW, Yeo IW (2000). Fluid Flow in Rock Fractures: From the Navier-Stokes Equations to the Cubic Law. In: Faybishenko B, Witherspoon PA, Benton SM, eds. Dynamics of Fluids in Fractured Rock. AGU Geophys Mono 122:213-24. 